\title{
Influence of Shape Variation on Capacitance Matrices
}

\author{
Daniël De Zutter, Dries Vande Ginste \\ Information Technology Department, Ghent University, St. Pietersnieuwstraat 41, 9000 \\ Ghent, Belgium.
}

\begin{abstract}
We theoretically determine the per-unit-of-length $N \times N$ capacitance matrix of a set of $N$ conductors w.r.t. a reference conductor, obtained when expanding the cross-section of one or more of these conductors w.r.t. some nominal configuration. It is shown that certain relationships between the individual matrix elements of the nominal and of the expanded configuration exist. For the $N \leq 2$ case, the expansion leads to the increase of the absolute value of all matrix elements. For $N>2$ no such general conclusion is shown to exist. The results remain valid in three dimensions. A number of numerical examples illustrate the theory.
\end{abstract}

Keywords: Capacitance matrix, shape variation.

\section{Introduction}

It has been known for a long time that the capacitance of a three-dimensional (3D) conductor is a slowly changing function of the conductor shape for a constant conductor surface area [1],[2]. This of course also applies to the twodimensional (2D) case replacing the capacitance by its per-unit-of-length (p.u.l.) value and the surface of the conductor by the circumference of its $2 \mathrm{D}$ crosssection. The introduction of a so-called shape factor in [1],[2] was particularly useful to estimate the capacitance of arbitrarily shaped conductors when fast computations were still lacking but is now of a rather archival interest. However, the knowledge that the capacitance is governed by some bounds has later on been used in various contexts [3],[4],[5].

In this paper, the influence of the conductor's shape will be revisited from an altogether different perspective. When considering an interconnection on a chip, inside a package, on a board, a backplane or via a cable, this interconnection is often modeled as part of a multiconductor transmission line. To this end the p.u.l. resistance, inductance, conductance and capacitance (RLGC) matrices have to be determined based on a quasi-TEM or, more generally, quasi-TM,

Email addresses: daniel.dezutter@ugent.be (Daniël De Zutter), dries.vandeginste@ugent.be (Dries Vande Ginste) 
description of the electromagnetic field problem [6]. The capacitance matrix follows from the solution of Poisson's equation in the cross-section. For highfrequencies (i.e. when the skin-effect is fully developed) the inductance matrix is directly proportional to the inverse of the so-called vacuum capacitance matrix [7]. This vacuum capacitance matrix again follows from the solution of Poisson's equation in the cross-section but with all (non-magnetic) dielectrics replaced by free space. Capacitance and inductance matrices determine the signal velocities and impedances of the modes that can propagate along an interconnection. Considering both the nominal design values and their variations due to manufacturing tolerances, it is tempting to extend the previously mentioned investigations into the shape dependence of the capacitance of a single conductor to this more general case and to ask whether anything can be proven about the influence of conductor shape changes on the values of the elements of the capacitance matrix. The theoretically established properties presented in this paper can be leveraged later on for the construction of fast algorithms for, e.g., the stochastic variability analysis of interconnect structures [8].

This paper is organized as follows. Section 2 presents the geometry of the problem and formulates the nominal capacitance problem (with p.u.l. capacitance matrix $\mathcal{C}$ ) together with its shape change counterpart (with modified p.u.l. capacitance matrix $\tilde{\mathcal{C}}$ ). Section 3 derives the general relationship existing between the two capacitance problems. As a starting point and for clarity, a two signal conductor system is considered. Section 4 derives conclusions as to the ratio of the individual elements of $\mathcal{C}$ and $\tilde{\mathcal{C}}$ and then extends the results to the general multiconductor case. Section 5 presents two sample numerical results and the conclusions are formulated in Section 6.

\section{Geometry of the problem}

Consider the cross-section of a 2D multiconductor transmission line (MTL) consisting of two signal lines and a reference conductor as depicted in Fig. 1.a. The z-axis is oriented along the longitudinal direction and the cross-section is invariant along this longitudinal direction. Although the analysis put forward below is readily extendible to a more general MTL consisting of $N$ signal conductors and a reference conductor, for clarity, we will restrict the detailed analysis in this section to the $N=2$ case. We suppose that all conductors are either perfectly conducting or are very good conductors (typically copper). They can be embedded in a lossless arbitrary dielectric background, either homogeneous or inhomogeneous but linear. We now focus our attention to the capacitance problem. Provided the conductors are either perfectly conducting or highly conducting and the dielectric losses are zero or remain very small [6], the formulation of the relevant capacitance problem, for the $2 \times 2$ problem of Fig. 1.a, is

$$
\begin{array}{r}
\mathbf{e}=-\nabla \phi \\
\nabla \cdot(\epsilon \mathbf{e})=0
\end{array}
$$




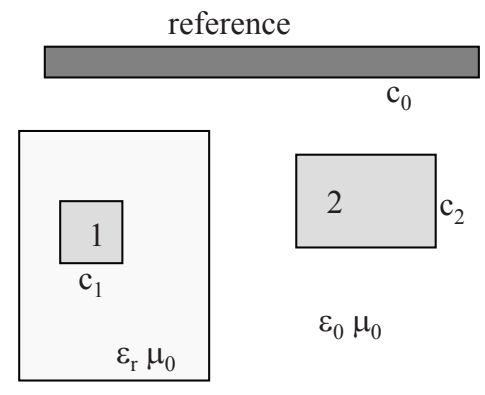

(a)

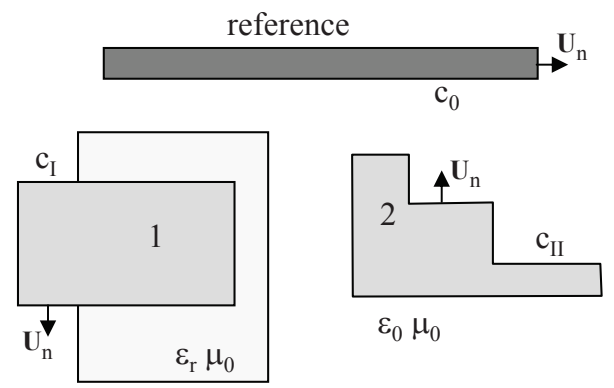

(b)

Figure 1: Nominal and modified configuration

with the potential $\phi$ satisfying

$$
\begin{array}{r}
\nabla^{2} \phi=0 \\
\phi=0 \text { on } c_{0} \\
\phi=v_{1} \text { on } c_{1} \\
\phi=v_{2} \text { on } c_{2}
\end{array}
$$

The boundaries $c_{1}, c_{2}$ and $c_{0}$ are the respective boundaries of the cross-sections of the signal lines and of the reference conductor. The relevant capacitance matrix $\mathcal{C}$ satisfies $\mathbf{q}=\mathcal{C} \mathbf{v}$, with $\mathbf{v}$ the column vector $\left[\begin{array}{ll}v_{1} & v_{2}\end{array}\right]^{T}$ formed by the constant potentials of the signal lines (more precisely, potential differences w.r.t. the reference conductor) and with $\mathbf{q}$ the column vector $\left[\begin{array}{ll}q_{1} & q_{2}\end{array}\right]^{T}$ formed by the total surface charge per unit of length (p.u.l.) on the signal lines. Hence, we have that

$$
\begin{aligned}
& q_{1}=C_{11} v_{1}+C_{12} v_{2} \\
& q_{2}=C_{12} v_{1}+C_{22} v_{2}
\end{aligned}
$$

By putting $C_{12}=C_{21}$ we have immediately used the general property that $\mathcal{C}$ is symmetric. Moreover, it can be proven for the general $N \times N$ case that $\mathcal{C}$ 
is not only symmetric but also positive definite, that all diagonal elements are positive and that all non-diagonal elements are negative [7]. We will use these properties in the sequel.

Let us now turn to the topic of our investigation by expanding the signal conductors from their nominal cross-section to a larger cross-section as shown in Fig. 1.b. The reference conductor is left unchanged and the expansion is such that the signal conductors do not touch. The background dielectrics also remain unchanged (not withstanding the part which is now taken up by the expanding conductors). The boundaries of the new cross-section are denoted as $c_{I}$ and $c_{I I}$ and the new configuration is described by the following equations, similar to (1)-(5):

$$
\begin{array}{r}
\mathbf{E}=-\nabla \Phi \\
\nabla \cdot(\epsilon \mathbf{E})=0
\end{array}
$$

with the potential $\Phi$ now satisfying

$$
\begin{array}{r}
\nabla^{2} \Phi=0 \\
\Phi=0 \text { on } c_{0} \\
\Phi=V_{1} \text { on } c_{I} \\
\Phi=V_{2} \text { on } c_{I I}
\end{array}
$$

The new capacitance matrix $\tilde{\mathcal{C}}$ satisfies $\mathbf{Q}=\tilde{\mathcal{C}} \mathbf{V}$ with

$$
\begin{aligned}
& Q_{1}=\tilde{C}_{11} V_{1}+\tilde{C}_{12} V_{2} \\
& Q_{2}=\tilde{C}_{12} V_{1}+\tilde{C}_{22} V_{2}
\end{aligned}
$$

The question now arises if it is possible to make any statement about the ratio of the elements in the two capacitance matrices $\mathcal{C}$ and $\tilde{\mathcal{C}}$. This will be treated in the next section.

\section{Relationship between the nominal and the modified capacitance matrix}

To start, multiplication of (2) with $\Phi$, of (7) with $\phi$ and subtracting, yields

$$
\Phi \nabla \cdot(\epsilon \mathbf{e})-\phi \nabla \cdot(\epsilon \mathbf{E})=0
$$

This can be further transformed into

$$
[\nabla \cdot(\epsilon \Phi \mathbf{e})-\epsilon \mathbf{e} \cdot \nabla \Phi]-[\nabla \cdot(\epsilon \phi \mathbf{E})-\epsilon \mathbf{E} \cdot \nabla \phi]=0
$$

Using (1) and (6), the second and fourth term cancel out. Next, the remaining divergences are integrated over the surface enclosed by boundaries $c_{0}, c_{I}$ and $c_{I I}$ of Fig. 1.b, yielding

$$
\int_{c_{0}+c_{I}+c_{I I}} \Phi\left(\mathbf{U}_{n} \cdot \mathbf{d}\right) d c=\int_{c_{0}+c_{I}+c_{I I}} \phi\left(\mathbf{U}_{n} \cdot \mathbf{D}\right) d c
$$


where we have now introduced the dielectric displacement vectors $\mathbf{D}=\epsilon \mathbf{E}$ and $\mathbf{d}=\epsilon \mathbf{e}$ and with $\mathbf{U}_{n}$ the outward pointing unit vectors as indicated in Fig. 1.b. As $\phi$ and $\Phi$ are zero on $c_{0}$, the contributions of $c_{0}$ in (13) drop out. Using (8), the l.h.s. of (13) yields

$$
V_{1} \int_{c_{I}}\left(\mathbf{U}_{n} \cdot \mathbf{d}\right) d c+V_{2} \int_{c_{I I}}\left(\mathbf{U}_{n} \cdot \mathbf{d}\right) d c
$$

Next, we turn back to $(2)$, i.e. $\nabla \cdot \mathbf{d}=0$, and integrate this identity over the surface bounded by $c_{1}$ and $c_{I}$, see shaded area of Fig. 2 . With $\mathbf{u}_{n}$ the outward pointing normal to $c_{1}$, we have that

$$
\int_{c_{I}}\left(\mathbf{U}_{n} \cdot \mathbf{d}\right) d c=\int_{c_{1}}\left(\mathbf{u}_{n} \cdot \mathbf{d}\right) d c=q_{1}
$$

with the total p.u.l. surface charge $q_{1}$ as given by (4). Similarly,

$$
\int_{c_{I I}}\left(\mathbf{U}_{n} \cdot \mathbf{d}\right) d c=\int_{c_{2}}\left(\mathbf{u}_{n} \cdot \mathbf{d}\right) d c=q_{2}
$$

with $q_{2}$ as given by (5). Using (4), (5), (15) and (16), (14) becomes

$$
C_{11} v_{1} V_{1}+C_{12}\left(v_{1} V_{2}+v_{2} V_{1}\right)+C_{22} v_{2} V_{2}=\mathbf{V}^{T} \mathcal{C} \mathbf{v}=\mathbf{v}^{T} \mathcal{C} \mathbf{V}
$$

Let us now return to the r.h.s. of (13). This can be rewritten as

$$
\int_{c_{I}} \phi \varrho_{I} d c+\int_{c_{I I}} \phi \varrho_{I I} d c
$$

with $\varrho_{I}$ and $\varrho_{I I}$ the p.u.l. surface charge densities on $c_{I}$ and $c_{I I}$ resp., pertaining to the potential problem (6)-(10) for the modified configuration.

Up to this point, no specific values for $v_{1}, v_{2}, V_{1}$ and $V_{2}$ have been introduced. To derive relationships between the elements of $\mathcal{C}$ and $\tilde{\mathcal{C}}$, we now select particular values for these potentials. By way of example, let us assign the following values: $v_{1}=1, v_{2}=0, V_{1}=1, V_{2}=0$. For these values, (17) reduces to $C_{11}$. For the particular potential problems we are now considering, we know that for any arbitrary place vector $\mathbf{r}$ in the cross-section we have that $0 \leq \phi \leq 1$. Also note that for $V_{1}=1$ and $V_{2}=0, \varrho_{I}>0$ and $\varrho_{I I}<0$. In the first term of (18), along $c_{I}, \phi$ will reach a certain maximum value. This maximum value will however always remain smaller than one. Hence,

$$
\int_{c_{I}} \phi \varrho_{I} d c=A Q_{1} \leq \int_{c_{I}} \varrho_{I} d c=Q_{1}
$$

with $0 \leq A \leq 1$ and $Q_{1}=\tilde{\mathcal{C}}_{11}$. Remark that, in contrast to $\phi, A$ is now a fixed number which of course still depends on the precise location of contour $c_{I}$. A similar reasoning for the second term in (18) gives

$$
-\int_{c_{I I}} \phi \varrho_{I I} d c=B\left|Q_{2}\right| \leq \int_{c_{I I}}\left|\varrho_{I I}\right| d c=\left|Q_{2}\right|
$$




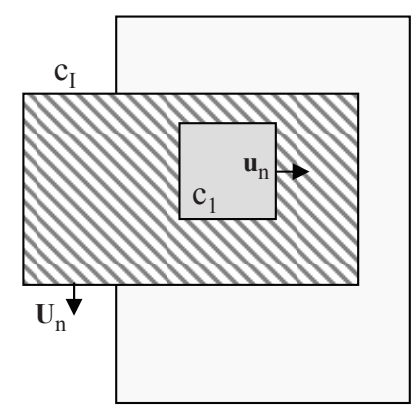

Figure 2: Integration of $\nabla \cdot \mathbf{d}$ over the shaded area.

with $0 \leq B \leq 1$ and $\left|Q_{2}\right|=\left|\tilde{\mathcal{C}}_{12}\right|$. In deriving (20) we have taken into account that $\varrho_{I I}<0$. Via (17) and (18), and for the selected values of the potentials, (13) leads to

$$
C_{11}=A \tilde{C}_{11}-B\left|\tilde{C}_{12}\right|
$$

Further note that, as the cross-sections of the expanded conductors do not intersect, we must have that $A \geq B$. This is a consequence of the fact that under these circumstances all potential values on $c_{I}$ are larger than those on $c_{I I}$. Similar results can now be derived by selecting other values for $v_{1}, v_{2}, V_{1}$ and $V_{2}$, e.g. for $v_{1}=0, v_{2}=1, V_{1}=1, V_{2}=0$ a similar results as above will be obtained for $C_{12}$. These results are discussed in more detail in the next section.

\section{Relationships between the elements of $\mathcal{C}$ and $\tilde{\mathcal{C}}$}

For $N=1$, i.e. a single signal line and a reference conductor, from (21) and with the second term in the r.h.s not present, the trivial result that the p.u.l. capacitance of the expanded signal conductor is higher than its nominal value is obtained.

For $N=2$, the approach put forward above implies that

$$
\begin{array}{r}
C_{11}=A \tilde{C}_{11}-B\left|\tilde{C}_{12}\right| \\
\left|C_{12}\right|=-C \tilde{C}_{11}+D\left|\tilde{C}_{12}\right| \\
C_{22}=E \tilde{C}_{22}-F\left|\tilde{C}_{12}\right|
\end{array}
$$

where $A$ to $F$ are constants ranging between zero and one and with $A \geq B, C \leq$ $D, E \geq F$. Relationships (22)-(24) proof that

$$
\begin{array}{r}
C_{11} \leq \tilde{C}_{11} \\
\left|C_{12}\right| \leq\left|\tilde{C}_{12}\right| \\
C_{22} \leq \tilde{C}_{22}
\end{array}
$$


i.e. all elements of the $2 \times 2$ capacitance matrix (with the proper sign convention) increase when the signal conductors expand.

A special case of practical interest is that of a symmetric differential line pair for which $C_{11}=C_{22}$. When the symmetry is retained while the signal conductors expand, $A=E$ and $B=F$ and of course $\tilde{C}_{11}=\tilde{C}_{22}$.

Turning to the general $N \times N$ case, one would hope to be able to derive similar properties as in the $2 \times 2$ case. We must however partly disappoint the reader. Let us first look at the equivalent of $(22)$ for the $3 \times 3$ case:

$$
C_{11}=A \tilde{C}_{11}-B\left|\tilde{C}_{12}\right|-C\left|\tilde{C}_{13}\right|
$$

with $A, B$ and $C$ of course different from the symbols used in the $2 \times 2$ case, but still $0 \leq A, B, C \leq 1$. It is clear that (28) readily implies that $C_{11} \leq \tilde{C}_{11}$ and this result is easily generalized to

$$
C_{i i} \leq \tilde{C}_{i i} i=1,2, \ldots, N
$$

generalizing (25) and (27). For off-diagonal elements however, e.g. for $N=3$, the expression for $\left|C_{12}\right|$ becomes

$$
\left|C_{12}\right|=-D \tilde{C}_{11}+E\left|\tilde{C}_{12}\right|+F\left|\tilde{C}_{13}\right|
$$

with $0 \leq D, E, F \leq 1$. Due to the positive term $F\left|\tilde{C}_{13}\right|$, one can no longer draw any conclusion as to the ratio of $\left|\tilde{C}_{12}\right|$ to $\left|C_{12}\right|$, or more generally

$$
C_{i j} \leq \tilde{C}_{i j} \text { or } C_{i j} \geq \tilde{C}_{i j}
$$

for $i \neq j$. In Section 5 a numerical example will be given confirming the above conclusions for the $3 \times 3$ case.

At this point it is appropriate to remark that the proof presented in Section 3 and the ensuing properties for the individual capacitance matrix elements remain completely valid in the three-dimensional case.

Before turning to the numerical examples, two further points, raised by the reviewers, merit our attention. One reviewer wondered what happens in the situation of Fig. 1 when the first conductor expands while the second one contracts. Close scrutiny of the reasoning put forward in Section 3 reveals that in this case no conclusive results can be derived. For this situation, the line of reasoning breaks down in (16) as this part of the proof no longer holds. A second reviewer views result (29) in the light of electrostatic energy density. Suppose that all conductors except conductor $i$ are put to zero potential while conductor $i$ is kept on unit potential. The fact that $\tilde{C}_{i i} \geq C_{i i}$ implies that the electrostatic energy $\frac{1}{2} \tilde{C}_{i i}^{2}=\frac{1}{2} \int \mathbf{E} \cdot \mathbf{D}$ of the expanded configuration is higher than the original one $\frac{1}{2} C_{i i}^{2}=\frac{1}{2} \int \mathbf{e} \cdot \mathbf{d}$. In both integrals, the integration extends over entire the space between the conductors.

\section{Numerical examples}

Although in principle it suffices to point out that a rigorous proof of the effect of shape change on the elements of the capacitance matrix has been 


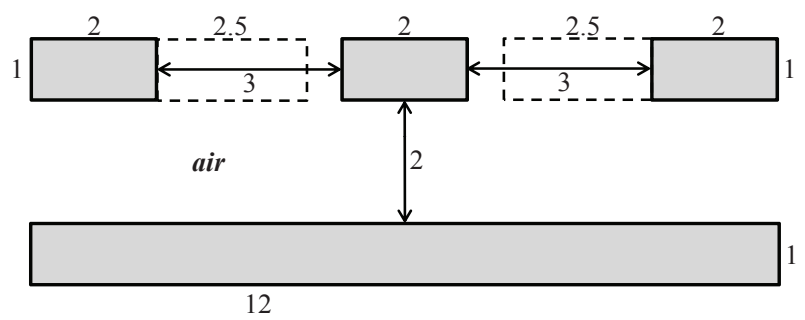

Figure 3: Three signal conductor example (all units in $\mu m$ )

given above, we will illustrate this proof with two examples. The first example, the initial cross-section of which is shown in Fig. 3, is a three signal conductor configuration in free space. All conductors are perfectly conducting. The three signal conductors have an identical cross-section of $2 \mu \mathrm{m} \times 1 \mu \mathrm{m}$ and the spacing between them is $3 \mu \mathrm{m}$. The reference (ground) conductor has a $12 \mu m \times 1 \mu m$ cross-section and the distance between the signal conductors and the reference conductor is $2 \mu \mathrm{m}$. The elements of the p.u.l. capacitance matrix for this initial configuration are: $C_{11}=C_{33}=30.4946 \mathrm{pF} / \mathrm{m}, C_{22}=34.9289 \mathrm{pF} / \mathrm{m}$, $C_{12}=C_{21}=C_{23}=C_{32}=-7.1541 p F / m, C_{13}=-1.9324 p F / m$. These values were obtained using the integral equation methods described in [6] and [9]. Of course other numerical techniques can be used to obtain these capacitances, see e.g. [10]. The numbering of the signal conductors is from the left to the right. The reader should remark that we have, on purpose, not selected an exotic cross-section but a rather banal one. First, we let the outer right conductor (conductor 3) expand in width to the left in 50 steps of $0.05 \mu \mathrm{m}$ (the final position is indicated in the figure by the dashed lines). Next, the outer left conductor (conductor 1) expands in width to the right, again in 50 steps of $0.05 \mu \mathrm{m}$. In the final situation, the configuration is again fully symmetrical and the corresponding elements of the p.u.l. capacitance matrix now are: $C_{11}=C_{33}=63.0925 \mathrm{pF} / \mathrm{m}, C_{22}=72.8476 \mathrm{pF} / \mathrm{m}$, $C_{12}=C_{21}=C_{23}=C_{32}=-30.4811 p F / m, C_{13}=-3.6834 p F / m . \quad$ Fig. 4 displays the evolution of $C_{i j r}=C_{i j}(n) / C_{i j}(1)$ for $n=1$ to $n=101$, i.e. the two times 50 positions plus the initial one. Remark that $C_{12 r}=C_{21 r}$ first decreases, to reach a minimum value of 0.9305 , to start increasing when the right conductors has reached its end position and the left one starts growing. A similar evolution is found for $C_{23 r}=C_{32 r}$, but here its value first increases, to reach a maximum of 4.3697 . On the other hand $C_{13}=C_{31}$ increases monotonically. These results clearly show that no definite statement can be made about the variation of the coupling capacitances $C_{i j}(i \neq j)$. The same example shows that all $C_{i i}$ increase monotonically. Looking at $C_{11}$, it is evident that expanding the right conductor only marginally effects its value $\left(C_{11 r}\right.$ increases from 1 to 1.00098). A similar remarks holds for $C_{33}$, the increase of which is only marginally affected by expanding the left conductor $\left(C_{33 r}\right.$ increases from 2.0669 to 2.0690$)$.

As a second example a two signal conductor problem is presented (Fig. 5). This 


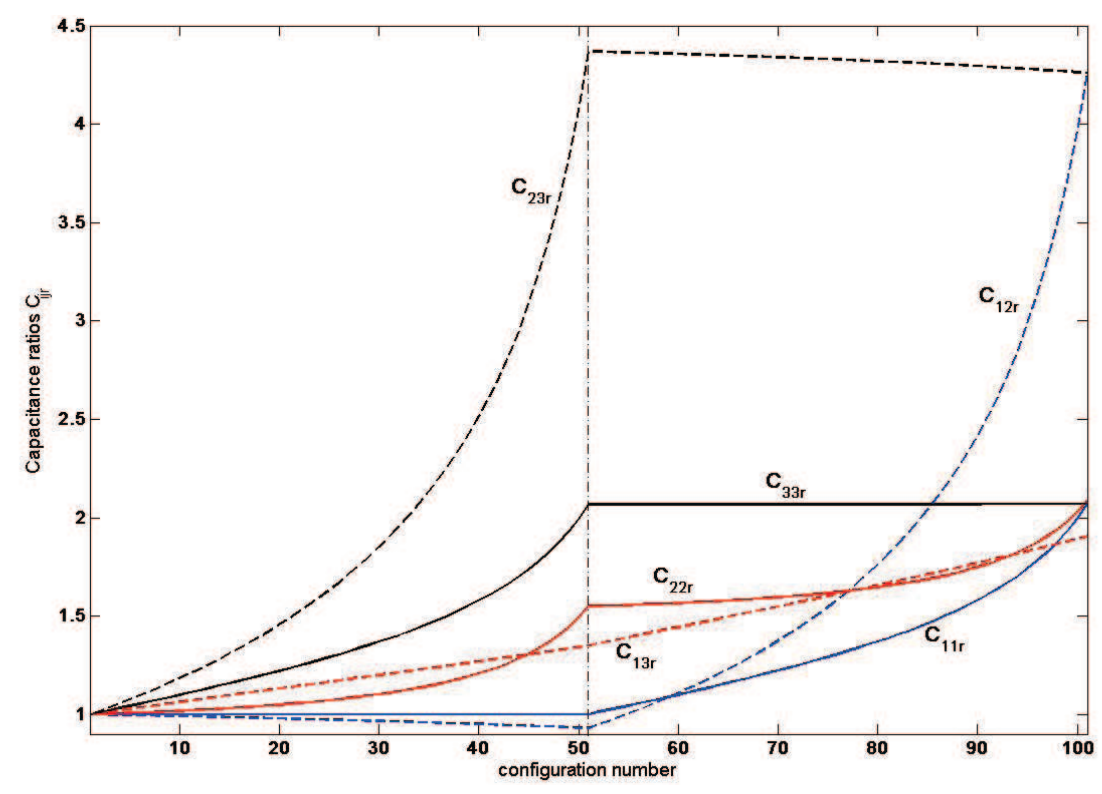

Figure 4: Relative variation of $C_{i j}$ as a function of expanding the left and right signal conductor (positions 1-51: first, expanding right conductor only; positions 52-101: next, expanding left conductor)

problem has a geometry which is quite similar to the one of the first example. However, the middle conductor is left out and a dielectric with relative dielectric permittivity of $\varepsilon_{r}=10$ is inserted underneath the right conductor (conductor 2) and occupies the whole space between the conductor and the reference conductor. We now let the right conductor expand towards the reference conductor (i.e. its height is changed, keeping the top side fixed and moving the bottom side in 30 steps of $0.04 \mu \mathrm{m}$ ). While doing so, the gap (which hence changes from an initial $2 \mu \mathrm{m}$ to a final $0.8 \mu \mathrm{m}$ ) between the bottom of the signal conductor and the ground plane remains filled with dielectric. The reasoning behind this particular example is that one might expect the electric field lines to be concentrated underneath the right conductor as such preventing $\left|C_{12}\right|$ and/or $C_{11}$ to increase. Fig. 6 presents the relative change of $C_{11}, C_{12}=C_{21}$ and $C_{22}$ as a function of the expansion of the right conductor. The initial p.u.l. capacitances are: $C_{11}=28.6619 \mathrm{pF} / \mathrm{m}, C_{22}=108.7754 \mathrm{pF} / \mathrm{m}, C_{12}=C_{21}=-3.3010 \mathrm{pF} / \mathrm{m}$. As predicted by the theory, $C_{12 r}$ and $C_{22 r}$ clearly increase with $1 \leq C_{12 r} \leq 1.0815$ and $1 \leq C_{22 r} \leq 2.2990$. Viewed on the same scale, $C_{11 r}$ seems constant, but its, albeit very small, increase is made visible in the bottom curve of Fig. 6 , where we have plotted 500 times $\Delta C_{11}$, i.e. the difference between $C_{11}$ for each configuration and the starting value of 28.6619 (expressed in $\mathrm{pF} / \mathrm{m}$ ). This corresponds to $C_{11 r}$ increasing from 1 to 1.00004 . In this example we have taken care to use many discretisation points in our code in order to obtain sufficiently accurate results. Hence, the above example confirms the general assertions (25)-(27). 


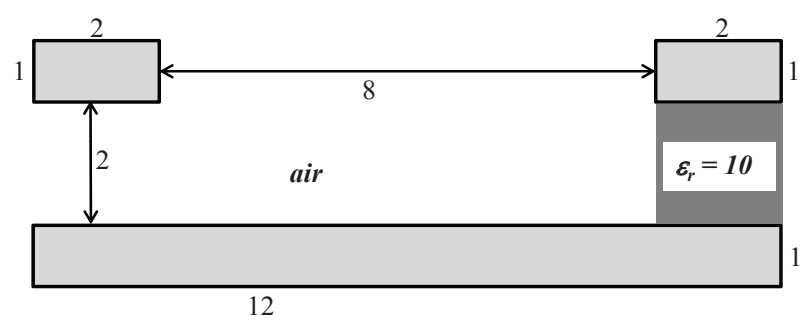

Figure 5: Two signal conductor example (all units in $\mu m$ )

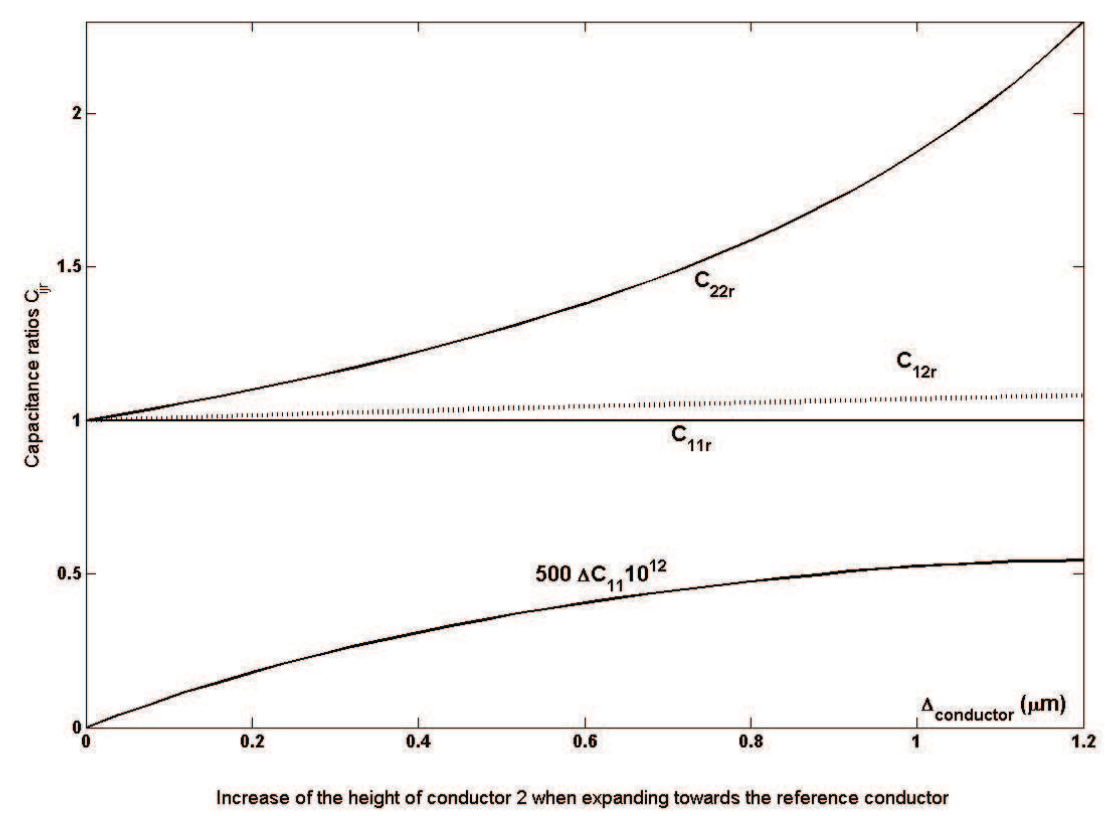

Figure 6: Relative variation of $C_{i j}$ as a function of increasing the height of the left conductor from $1 \mu m$ to $2.2 \mu m$. 


\section{Conclusions}

In this paper it has been investigated how the elements of the p.u.l. $N \times$ $N$ capacitance matrix of a system of $N$ signal conductors w.r.t. a reference conductor change when the cross-section of one or more of the signal conductors expands w.r.t. to the nominal, i.e. originally considered, geometry. It turned out to be possible to rigorously prove that for $N=1$ and $N=2$, the absolute value of these element values (the $\mathrm{C}$-value in the $N=1$ case and $C_{11},\left|C_{12}\right|=\left|C_{21}\right|$ and $C_{22}$ in the $N=2$ case) will always increase monotonically. An example was added to support the theoretical claim in a case where this claim is not clear in a straightforward way.

However, for $N>2$, it was shown that such assertions are not possible and that the only property that still holds is that the diagonal elements $C_{i i}$ will increase. For the non-diagonal elements $\left|C_{i j}\right|$ either an increase or a decrease is possible and when considering a particular case, these non-diagonal elements can first decrease when expanding a cross-section to start increasing when the crosssection further expands (or vice-versa), as demonstrated with an illustrative example.

\section{References}

[1] Y.L. Chow, M.M. Yovanovich, The shape factor of the capacitance of a conductor, Journal of Applied Physics 53 (1982) 8470-8475.

[2] G. Crasta, I. Fragalà, F. Gazzola, On a long-standing conjecture by PólyaSzegö and related topics, Z. angew. Math. Phys. 56 (2005) 763-782.

[3] M. Ouda, A.-R. Sebak, Minimizing the computational cost and memory requirement for the capacitance calculation of 3-D multiconductor systems, IEEE Trans. Components, Packaging and Manufacturing Techn. - Part A 18 (1995) 685-689.

[4] Y. L. Chow, W. C. Tang, Development of CAD formulas of integrated circuit components: fuzzy EM formulation followed by rigorous derivation, Journal of Electromagentic Waves and Applications 15 (2001) 1097-1119.

[5] S.-T. Kwok, K.-F. Tsang, Y. L. Chow, Capacitance of a dielectric post separating a conducting patch above the air-ground, Microwave and Optical Technology Letters 42 (2004) 441-444.

[6] T. Demeester, D. De Zutter, Quasi-TM transmission line parameters of coupled lossy lines based on the Dirichlet to Neumann boundary operator, IEEE Transactions on Microwave Theory and Techniques 56 (2008) 16491660 .

[7] W.-K. Chen edit., Circuits and Filters Handbook, Chapter 16: Multiconductor Transmission Lines, 3rd Edition, CRC Press, 2009. 
[8] D. Vande Ginste, D. De Zutter, D. Deschrijver, T. Dhaene, P. Manfredi, F. Canavero, Stochastic modeling based variability analysis of on-chip interconnects, IEEE Transactions on Components, Packinging, and Manufacturing Technology 2 (2012) 1182-1192.

[9] D. De Zutter, L. Knockaert, Skin effect modeling based on a differential surface admittance operator, IEEE Transactions on Microwave Theory and Techniques 53 (2005) 2526-2538.

[10] A.N. Racasan, C. Pacurar, C. Munteanu, V. Topa, D.D. Micu, C. Hebedean, Modeling and mitigation techniques of the magnetic integrated structures parasitic capacitance, Proc. of the 47th International Universities Power Engineering Conference, UPEC 2012 (2012) 1-5. 